\title{
Optical Soliton Perturbation in Nonlinear Directional Couplers
}

\author{
J. VeGa-Guzman ${ }^{a}$, M.M. Babatin ${ }^{b}$ And A. BisWas ${ }^{b, c, *}$ \\ ${ }^{a}$ Department of Mathematics, Lamar University, Beaumont, TX-77705, USA \\ ${ }^{b}$ Department of Mathematics and Statistics, College of Science, Al-Imam Mohammad Ibn Saud Islamic University, \\ Riyadh-13318, Saudi Arabia \\ ${ }^{c}$ Department of Mathematics and Statistics, Tshwane University of Technology, Pretoria-0008, South Africa
}

(Received July 2, 2017)

This paper secures soliton solutions to optical couplers in presence of Hamiltonian perturbation terms by the aid of undetermined coefficients. Both twin core couplers and multiple core couplers are studied. Bright, dark and singular soliton solutions are obtained for the model. The existence criteria for the solitons are also presented. The study is focused to the Kerr and power laws of nonlinearity.

DOI: 10.12693/APhysPolA.133.167

PACS/topics: solitons, couplers, metamaterials

\section{Introduction}

Optical solitons are visible in various kinds of devices in the field of nonlinear optics. Some of the most common areas of visibility are optical fibers, couplers, PCF, metamaterials and metasurfaces, DWDM systems and several others $[1-20]$. There are several results that are reported in the context of solitons in optical fibers. This paper will focus on the dynamics of solitons in optical couplers. There has been quite a few results that are visible in this area $[1,2,4,6-13,15,16,19]$. However, this paper retrieves exact soliton solutions to optical couplers in presence of Hamiltonian perturbation terms by the method of undetermined coefficients. There are two forms of nonlinear media that are studied in this paper. They are Kerr law and power law nonlinearities. Bright, dark and singular soliton solutions are extracted using this integration scheme. The existence criteria of these solitons are also presented which are labeled as constraint conditions. The details follow through in subsequent sections.

\section{Twin-core couplers}

Optical nonlinear couplers have been very useful devices to distribute light from a main fiber into one or more branch fibers. Couplers also have applications as intensity-dependent switches and as limiters. They can be used to multiplex two incoming bit streams onto a fiber and also to demultiplex a single-bit stream. The general model that govern the dynamics of nonlinear directional couplers with constant coefficients is given by:

$$
\begin{aligned}
& \mathrm{i} q_{t}+\mathrm{i} \alpha_{1} q_{x}+a_{1} q_{x x}+b_{1} q_{x t}+F\left(|q|^{2}\right) q \\
& \quad+\mathrm{i} \Gamma_{1} q_{x x x}+\mathrm{i} \lambda_{1}\left(|q|^{2} q\right)_{x}+\mathrm{i} \xi_{1}|q|^{2} q_{x} \\
& \quad+\mathrm{i} \eta_{1}\left(|q|^{2}\right)_{x} q=k_{1} r
\end{aligned}
$$

*corresponding author; e-mail: biswas.anjan@gmail.com

$$
\begin{aligned}
& \mathrm{i} r_{t}+\mathrm{i} \alpha_{2} r_{x}+a_{2} r_{x x}+b_{2} r_{x t}+F\left(|r|^{2}\right) r \\
& \quad+\mathrm{i} \Gamma_{2} r_{x x x}+\mathrm{i} \lambda_{2}\left(|r|^{2} r\right)_{x}+\mathrm{i} \xi_{2}|r|^{2} r_{x} \\
& \quad+\mathrm{i} \eta_{2}\left(|r|^{2}\right)_{x} r=k_{2} q
\end{aligned}
$$

Here $k_{j}$ for $j=1,2$ are coupling coefficients. Also, in (1) and $(2)$, the first terms are linear evolutions, while the coefficients $a_{j}$ and $b_{j}$, for $j=1,2$, gives group velocity dispersion (GVD) and spatio-temporal dispsersion (STD) respectively. The functional $F$ is the dependence on the generalized form of the refractive index of light pulses. Again, $\lambda_{j}$ account for self-steepening effect while $\xi_{j}$ and $\eta_{j}$ represent nonlinear dispersions. Finally, $\Gamma_{j}$ provides third order dispersion effect that is considered when GVD and STD are negligbly small.

We assume solution hypothesis to be of the form

$$
\begin{aligned}
& q(x, t)=P_{1}(x, t) \mathrm{e}^{\mathrm{i} \phi(x, t)} \\
& r(x, t)=P_{2}(x, t) \mathrm{e}^{\mathrm{i} \phi(x, t)}
\end{aligned}
$$

where $P_{l}(x, t)(l=1,2)$ represents the amplitude component of the soliton solution while the phase part $\phi(x, t)$ is defined as

$$
\phi(x, t)=-\kappa x+\omega t+\Theta .
$$

Here $\kappa$ is the frequency of the solitons while $\omega$ represents the wave number, and $\Theta$ the phase constant. Substituting (3) and (4) into (1) and (2) and then decomposing into real and imaginary components gives in a simplified form

$$
\begin{aligned}
& \left(a_{l}+3 \kappa \Gamma_{l}\right) \frac{\partial^{2} P_{l}}{\partial x^{2}}+b_{l} \frac{\partial^{2} P_{l}}{\partial x \partial t} \\
& \quad+\left[\kappa\left(\alpha_{l}+b_{l} \omega_{l}-a_{l} \kappa-\Gamma_{l} \kappa^{2}\right)-\omega\right] P_{l} \\
& \quad+\kappa\left(\xi_{l}+\lambda_{l}\right) P_{l}^{3}+F\left(P_{l}^{2}\right) P_{l}=k_{l} P_{\bar{l}}
\end{aligned}
$$

and

$$
\begin{gathered}
\left(1-b_{l} \kappa\right) \frac{\partial P_{l}}{\partial t}+\left(b_{l} \omega+\alpha_{l}-2 a_{l} \kappa-3 \Gamma_{l} \kappa^{2}\right) \frac{\partial P_{l}}{\partial x} \\
+\left(2 \eta_{l}+\xi_{l}+3 \lambda_{l}\right) P_{l}^{2} \frac{\partial P_{l}}{\partial x}+\Gamma_{l} \frac{\partial^{3} P_{l}}{\partial x^{3}}=0
\end{gathered}
$$

respectively, with $l=1,2$ and $\bar{l}=3-l$. It is well known that the profile function $P_{l}(x, t)$ can be written in the 
form $f(x-\nu t)$ where the function $f$ represents the soliton wave profile depending the type of nonlinearity, and $\nu$ the soliton speed. From the imaginary part (7) the soliton speed can be obtain as

$$
\nu=\frac{b_{l} \omega+\alpha_{l}-2 a_{l} \kappa}{1-b_{l} \kappa} \text {. }
$$

after the solvability conditions

$$
\Gamma_{l}=0
$$

and

$$
2 \eta_{l}+\xi_{l}+3 \lambda_{l}=0 .
$$

are imposed. By comparing both resulting values of the soliton speed one can conclude that the coefficients of the general system (1)-(2) become

$$
a_{1}=a_{2}, \quad b_{1}=b_{2} \quad \text { and } \quad \alpha_{1}=\alpha_{2} .
$$

In view of (11), the speed (8) is rewritten as

$$
\nu=\frac{\alpha+b \omega-2 a \kappa}{1-b \kappa} \text {. }
$$

regardless the nonlinearity under consideration, while the real portion (6), takes the following modified form:

$$
\begin{aligned}
& a \frac{\partial^{2} P_{l}}{\partial x^{2}}+b \frac{\partial^{2} P_{l}}{\partial x \partial t}+\{\kappa(\alpha+b \omega-a \kappa)-\omega\} P_{l} \\
& +\kappa\left(\xi_{l}+\lambda_{l}\right) P_{l}^{3}+F\left(P_{l}^{2}\right) P_{l}=k_{l} P_{\bar{l}}
\end{aligned}
$$

The real part equation (13) will be considered in the following subsections in view of two different forms of the functional $F\left(P_{l}\right)$, they are Kerr and power law nonlinearities.

\subsection{Kerr law nonlinearity}

When the Kerr law nonlinearity is considered $F(s)=s$, and the system (1)-(2) is rewritten as

$$
\begin{aligned}
& \mathrm{i} q_{t}+\mathrm{i} \alpha q_{x}+a q_{x x}+b q_{x t}+c_{1}|q|^{2} q+\mathrm{i} \lambda_{1}\left(|q|^{2} q\right)_{x} \\
& \quad+\mathrm{i} \xi_{1}|q|^{2} q_{x}+\mathrm{i} \eta_{1}\left(|q|^{2}\right)_{x} q=k_{1} r \\
& \mathrm{i} r_{t}+\mathrm{i} \alpha r_{x}+a r_{x x}+b r_{x t}+c_{2}|r|^{2} r+\mathrm{i} \lambda_{2}\left(|r|^{2} r\right)_{x} \\
& \quad+\mathrm{i} \xi_{2}|r|^{2} r_{x}+\mathrm{i} \eta_{2}\left(|r|^{2}\right)_{x} r=k_{2} q
\end{aligned}
$$

in view of (9)-(11). Consequently, the real component (13) takes the new form

$$
\begin{aligned}
& a \frac{\partial^{2} P_{l}}{\partial x^{2}}+b \frac{\partial^{2} P_{l}}{\partial x \partial t}+\{\kappa(\alpha+b \omega-a \kappa)-\omega\} P_{l} \\
& \quad+\left\{c_{l}+\kappa\left(\xi_{l}+\lambda_{l}\right)\right\} P_{l}^{3}=k_{l} P_{\bar{l}}
\end{aligned}
$$

where $l=1,2$ and $\bar{l}=3=l$. The next subsections will be devoted to solve (16) for bright, dark and singular (type-I and type-II) solitons.

\subsubsection{Bright solitons}

To explore the dynamics of bright soliton propagation in system (14)-(15), one solve the real part (16) with the aid of the starting hypothesis for $P_{l}$ :

$$
P_{l}=A_{l} \operatorname{sech}^{p_{l}} \tau
$$

where

$$
\tau=B(x-\nu t) .
$$

In (17) $A_{l}$ stands for the pulse amplitude while $B$ is the corresponding inverse width. The substitution of (17) into (16) leads to

$$
\begin{aligned}
& \left\{[\kappa(\alpha+b \omega-a \kappa)-\omega]+p_{l}^{2}(a-b \nu) B^{2}\right\} A_{l} \operatorname{sech}^{p_{l}} \tau \\
& \quad-k_{l} A_{\bar{l}} \operatorname{sech}^{p_{\bar{l}}} \tau \\
& \quad-p_{l}\left(1+p_{l}\right)(a-b \nu) A_{l} B^{2} \operatorname{sech}^{p_{l}+2} \tau \\
& \quad+\left[c_{l}+\kappa\left(\xi_{l}+\lambda_{l}\right)\right] A_{l}^{3} \operatorname{sech}^{3 p_{l}} \tau=0
\end{aligned}
$$

after simplification. Then, applying the balancing principle in (19) one obtain

$$
3 p_{l}=2+p_{l}
$$

leading to

$$
p_{l}=1
$$

for $l=1,2$. Setting the coefficients of the resulting linearly independent functions $\operatorname{sech}^{j} \tau$ to zero, for $j=1,3$ leads to the speed and wave numbers of the bright solitons as

$$
\nu=\frac{2 a B^{2}-\left\{c_{l}+\kappa\left(\lambda_{l}+\xi_{l}\right)\right\} A_{l}^{2}}{2 b B^{2}}
$$

and

$$
\omega=\frac{2 k_{l} A_{\bar{l}}-\left\{c_{l}+\kappa\left(\lambda_{l}+\xi_{l}\right)\right\} A_{l}^{3}-2 \kappa(\alpha-a \kappa) A_{l}}{2(b \kappa-1) A_{l}}
$$

respectively, as long as $b \kappa \neq 1$ and $b B \neq 0$. Moreover, one can notice by equating both alternative expressions for the soliton speed $\nu$ for $l=1,2$ in (21) the relation between the amplitudes

$$
\frac{A_{1}}{A_{2}}=\sqrt{\frac{c_{2}+\kappa\left(\lambda_{2}+\xi_{2}\right)}{c_{1}+\kappa\left(\lambda_{1}+\xi_{1}\right)}}
$$

constrained by

$$
\left\{c_{2}+\kappa\left(\lambda_{2}+\xi_{2}\right)\right\}\left\{c_{1}+\kappa\left(\lambda_{1}+\xi_{1}\right)\right\}>0 .
$$

Following a similar procedure, equating the two expressions for the soliton wave numbers from (22) for $l=1,2$ one obtain a connection between the amplitudes of bright solitons in the two components as

$$
\begin{aligned}
& 2 k_{1} A_{2}^{2}-\left\{c_{1}+\kappa\left(\lambda_{1}+\xi_{1}\right)\right\} A_{1}^{3} A_{2}=2 k_{2} A_{1}^{2} \\
& -\left\{c_{2}+\kappa\left(\lambda_{2}+\xi_{2}\right)\right\} A_{2}^{3} A_{1} .
\end{aligned}
$$

With the help of (23), by comparing (12) and (21) it is possible to obtain

$$
A_{l}=B \sqrt{-\frac{2\{a(1+b \kappa)-b(\alpha+b \omega)\}}{(b \kappa-1)\left\{c_{l}+\kappa\left(\lambda_{l}+\xi_{l}\right)\right\}}}
$$

for $l=1,2$ and $\bar{l}=3-l$ whenever the inequality $\{a(1+b \kappa)-b(\alpha+b \omega)\}(b \kappa-1)\left\{c_{l}+\kappa\left(\lambda_{l}+\xi_{l}\right)\right\}<0$.

holds. Also, by substituting (26) into (25) for both $l=1$ and $l=2$ one get two possible expressions for the frequency:

$$
\kappa=\frac{b(\alpha+b \omega)-a}{a b}
$$

and

$$
\kappa=\frac{c_{2} k_{2}-c_{1} k_{1}}{\left(\lambda_{1}+\xi_{1}\right) k_{1}-\left(\lambda_{2}+\xi_{2}\right) k_{2}}
$$


conditioned to

$$
a b \neq 0, \quad \frac{k_{1}}{k_{2}} \neq \frac{\left(\lambda_{2}+\xi_{2}\right)}{\left(\lambda_{1}+\xi_{1}\right)}
$$

respectively. Thus, we can conclude that the bright soliton solution for the system describing nonlinear directional couplers in optical metamaterials is given by

$$
\begin{aligned}
& q(x, t)=A_{1} \operatorname{sech}[B(x-\nu t)] \mathrm{e}^{\mathrm{i}(-\kappa x+\omega t+\Theta)} \\
& r(x, t)=A_{2} \operatorname{sech}[B(x-\nu t)] \mathrm{e}^{\mathrm{i}(-\kappa x+\omega t+\Theta)}
\end{aligned}
$$

where parameters and corresponding constraints are discussed above.

\subsubsection{Dark solitons}

To explore the propagation of dark solitons within the directional couplers (14)-(15), one integrate (16) with the ansatz

$$
P_{l}=A_{l} \tanh ^{p_{l}} \tau
$$

where the argument $\tau$ is defined as in (18), the pulse amplitude still describes by $A_{l}$, while $p_{l}$ is a free parameter to be determined throughout the next few lines. The substitution of (31) into (16) leads to

$$
\begin{aligned}
& \left\{[\kappa(\alpha+b \omega-a \kappa)-\omega]-2 p_{l}^{2}(a-b \nu) B^{2}\right\} A_{l} \tanh ^{p_{l}} \tau \\
& \quad+p_{l}\left(p_{l}-1\right)(a-b \nu) A_{l} B^{2} \tanh ^{p_{l}-2} \tau \\
& \quad+p_{l}\left(p_{l}+1\right)(a-b \nu) A_{l} B^{2} \tanh ^{p_{l}+2} \tau \\
& \quad+\left[c_{l}+\kappa\left(\xi_{l}+\lambda_{l}\right)\right] A_{l}^{3} \tanh ^{3 p_{l}} \tau \\
& \quad-k_{l} A_{\bar{l}} \tanh ^{p_{\bar{l}}} \tau=0
\end{aligned}
$$

The known balance between dispersion and nonlinearity, as well as the standalone linearly independent function $\tanh ^{p_{l}-2} \tau$ leads one to retrieve the value of $p_{l}$ as in (20). Thus, setting to zero the coefficients of the linearly independent functions $\tanh ^{j} \tau$ for $j=1,3$ leads to

$$
\nu=\frac{2 a B^{2}+\left\{c_{l}+\kappa\left(\lambda_{l}+\xi_{l}\right)\right\} A_{l}^{2}}{2 b B^{2}}
$$

and

$$
\omega=\frac{k_{l} A_{\bar{l}}-\left\{c_{l}+\kappa\left(\lambda_{l}+\xi_{l}\right)\right\} A_{l}^{3}-\kappa(\alpha-a \kappa)}{(b \kappa-1) A_{l}} .
$$

In (33), after comparing the two resulting waves speeds $v$ for $l=1,2$ the quotient between amplitudes implies the same relation as given in (23) and (24). Similarly, comparing the two resulting wave functions $\omega$ in (34) the following identity arise:

$$
\begin{aligned}
& k_{1} A_{2}^{2}+\left\{c_{1}+\kappa\left(\lambda_{1}+\xi_{1}\right)\right\} A_{1}^{3} A_{2}=k_{2} A_{1}^{2} \\
& \quad+\left\{c_{2}+\kappa\left(\lambda_{2}+\xi_{2}\right)\right\} A_{2}^{3} A_{1} .
\end{aligned}
$$

By considering (23) and comparing the two possible expressions for the speed (12) and (33) to each other for either value of $l$, yields

$$
A_{l}=B \sqrt{\frac{2\{a(1+b \kappa)-b(\alpha+b \omega)\}}{(b \kappa-1)\left\{c_{l}+\kappa\left(\lambda_{l}+\xi_{l}\right)\right\}}}
$$

as long as

$\{a(1+b \kappa)-b(\alpha+b \omega)\}(b \kappa-1)\left\{c_{l}+\kappa\left(\lambda_{l}+\xi_{l}\right)\right\}>0 .(37)$ stay valid. By substituting (36) into (35) for both $l=1$ and $l=2$ one recalculate $(28)-(30)$. Therefore, the dark soliton solution for the dispersive twin core couplers with cubic nonlinearity (14)-(15) is given by

$$
\begin{aligned}
& q(x, t)=A_{1} \tanh [B(x-\nu t)] \mathrm{e}^{\mathrm{i}(-\kappa x+\omega t+\Theta)} \\
& r(x, t)=A_{2} \tanh [B(x-\nu t)] \mathrm{e}^{\mathrm{i}(-\kappa x+\omega t+\Theta)}
\end{aligned}
$$

where the soliton amplitude is described by (36), the speed and the wave numbers are (33) and (34), while the frequency is either described by (28) or (29), with corresponding constraints.

\subsubsection{Singular solitons (type-I)}

In order to study the propagation dynamics of type-I singular soliton solutions, the waveform

$$
P_{l}=A_{l} \operatorname{csch}^{p_{l}} \tau \text {. }
$$

is adopted, where $A_{l}$ describes the pulse amplitude, the parameter $p_{l}$ will be calculated by balancing nonlinearity and dispersion, and the argument $\tau$ has been defined in (18). Direct substitution of (39) into (16) results in

$$
\begin{aligned}
& \{[ \\
& \left.\quad-(\alpha+b \omega-a \kappa)-\omega]+p_{l}^{2}(a-b \nu) B^{2}\right\} A_{l} \operatorname{csch}^{p_{l}} \tau \\
& \quad-k_{l} A_{\bar{l}} \operatorname{csch}^{p_{\bar{l}}} \tau \\
& \quad+p_{l}\left(1+p_{l}\right)(a-b \nu) A_{l} B^{2} \operatorname{csch}^{p_{l}+2} \tau \\
& \quad+\left[c_{l}+\kappa\left(\xi_{l}+\lambda_{l}\right)\right] A_{l}^{3} \operatorname{csch}^{3 p_{l}} \tau=0
\end{aligned}
$$

The balancing procedure enable to retrieve the value of the parameter $p_{l}$ as in (20). After substituting $p=1$, for $l=1,2$ in (40), setting the coefficients of the linearly independent functions $\operatorname{csch}^{j} \tau$ for $j=1,3$ to zero allow to retrieve the soliton speed as for dark soliton (33), while the wave numbers become

$$
\omega=\frac{2 k_{l} A_{\bar{l}}+\left\{c_{l}+\kappa\left(\lambda_{l}+\xi_{l}\right)\right\} A_{l}^{3}-2 \kappa(\alpha-a \kappa)}{2(b \kappa-1) A_{l}} .
$$

Equating the resulting two expressions for the soliton wave in the last equation (41) for $l=1,2$ gives

$$
\begin{aligned}
& 2 k_{1} A_{2}^{2}+\left\{c_{1}+\kappa\left(\lambda_{1}+\xi_{1}\right)\right\} A_{1}^{3} A_{2}=2 k_{2} A_{1}^{2} \\
& \quad+\left\{c_{2}+\kappa\left(\lambda_{2}+\xi_{2}\right)\right\} A_{2}^{3} A_{1} .
\end{aligned}
$$

Thus, considering (23) and equating the two possible expressions for the speed (12) and (33) to each other for either $l=1$ or $l=2$ yield (36) constrained by (37). Also substituting (23) and (33)) into (42) provides the same expressions for $\kappa$ and corresponding solvability conditions (28)-(30). Consequently, the type-I singular soliton solution for the system (14)-(15) is

$$
\begin{aligned}
& q(x, t)=A_{1} \operatorname{csch}[B(x-\nu t)] \mathrm{e}^{\mathrm{i}(-\kappa x+\omega t+\Theta)} \\
& r(x, t)=A_{2} \operatorname{csch}[B(x-\nu t)] \mathrm{e}^{\mathrm{i}(-\kappa x+\omega t+\Theta)} .
\end{aligned}
$$

This type-I singular soliton solution will persist whenever the corresponding constrains above discussed are satisfied.

\subsubsection{Singular solitons (type-II)}

To study the propagation dynamics of the second type of singular solitons within the system (14)-(15), we assume a solution of the form 


$$
P_{l}=A_{l} \operatorname{coth}^{p_{l}} \tau
$$

where the meaning of the amplitude $A_{l}$, the argument $\tau$ and the parameter $p_{l}$ is the same as for the previous starting hypothesis in Kerr nonlinearity. The substitution of the hypothesis (43) into (16) leads to

$$
\begin{aligned}
& \{[ \\
& \left.\quad(\alpha+b \omega-a \kappa)-\omega]-2 p_{l}^{2}(a-b \nu) B^{2}\right\} A_{l} \operatorname{coth}^{p_{l}} \tau \\
& \quad+p_{l}\left(p_{l}-1\right)(a-b \nu) A_{l} B^{2} \operatorname{coth}^{p_{l}-2} \tau \\
& \quad+p_{l}\left(p_{l}+1\right)(a-b \nu) A_{l} B^{2} \operatorname{coth}^{p_{l}+2} \tau \\
& \quad+\left[c_{l}+\kappa\left(\xi_{l}+\lambda_{l}\right)\right] A_{l}^{3} \operatorname{coth}^{3 p_{l}} \tau \\
& \quad-k_{l} A_{\bar{l}} \operatorname{coth}^{p_{\bar{l}}} \tau=0 .
\end{aligned}
$$

Balancing principle, as well as the coefficient of the stand alone element $\operatorname{coth}^{p_{l}-2} \tau$ yields the same value of the parameter $p_{l}$ as in (20). The substitution of the resulting value of $p_{l}$ into (44) leads to the same results as for dark solitons (33)-(37) along with corresponding conditions.

Therefore, the type-I singular soliton solution for the nonlinear system (14)-(15) is of the form

$$
\begin{aligned}
& q(x, t)=A_{1} \operatorname{coth}[B(x-\nu t)] \mathrm{e}^{\mathrm{i}(-\kappa x+\omega t+\Theta)} \\
& r(x, t)=A_{2} \operatorname{coth}[B(x-\nu t)] \mathrm{e}^{\mathrm{i}(-\kappa x+\omega t+\Theta)} .
\end{aligned}
$$

where the corresponding parameters as well as constraints are the same as for dark solitons.

\subsection{Power law nonlinearity}

This subsection is dedicated to investigate in detail the propagation of solitons in the system (1)-(2) under the influence of power law nonlinearity. In this case, the nonlinear functional has the general form $F(s)=s^{n}$ where $n$ represents the power law nonlinearity parameter. For wave stability purposes we are restricted to $0<n<2$, strictly imposing $n \neq 2$ to evade self-focusing singularity. A starting step is to rewrite the original system (1)-(2) as

$$
\begin{aligned}
& \mathrm{i} q_{t}+\mathrm{i} \alpha_{1} q_{x}+a_{1} q_{x x}+b_{1} q_{x t}+c_{1}|q|^{2 n} q \\
& \quad+\mathrm{i} \Gamma_{1} q_{x x x}+\mathrm{i} \lambda_{1}\left(|q|^{2 n} q\right)_{x}+\mathrm{i} \xi_{1}|q|^{2 n} q_{x} \\
& \quad+\mathrm{i} \eta_{1}\left(|q|^{2 n}\right)_{x} q=k_{1} r \\
& \mathrm{i} r_{t}+\mathrm{i} \alpha_{2} r_{x}+a_{2} r_{x x}+b_{2} r_{x t}+c_{2}|r|^{2 n} r \\
& \quad+\mathrm{i} \Gamma_{2} r_{x x x}+\mathrm{i} \lambda_{2}\left(|r|^{2 n} r\right)_{x}+\mathrm{i} \xi_{2}|r|^{2 n} r_{x} \\
& \quad+\mathrm{i} \eta_{2}\left(|r|^{2 n}\right)_{x} r=k_{2} q
\end{aligned}
$$

By substituting (3) and (4) into (45) and (46), the real component (13) is transformed into

$$
\begin{aligned}
& a_{l} \frac{\partial^{2} P_{l}}{\partial x^{2}}+b_{l} \frac{\partial^{2} P_{l}}{\partial x \partial t}+\left\{\kappa\left(\alpha_{l}+b_{l} \omega-a_{l} \kappa\right)-\omega-\Gamma_{l} \kappa^{3}\right\} P_{l} \\
& \quad+\left\{c_{l}+\kappa\left(\xi_{l}+\lambda_{l}\right)\right\} P_{l}^{2 n+1}=k_{l} P_{\bar{l}}
\end{aligned}
$$

while the corresponding imaginary part takes the form

$$
\left(1-b_{l} \kappa\right) \frac{\partial P_{l}}{\partial t}+\left(b_{l} \omega+\alpha_{l}-2 a_{l} \kappa-3 \Gamma_{l} \kappa^{2}\right) \frac{\partial P_{l}}{\partial x}
$$

$$
\begin{aligned}
& \left.+\left\{2 n \eta_{l}+\xi_{l}+(2 n+1) \lambda_{l}\right)\right\} P_{l}^{2} \frac{\partial P_{l}}{\partial x} \\
& +\Gamma_{l} \frac{\partial^{3} P_{l}}{\partial x^{3}}=0
\end{aligned}
$$

As for Kerr nonlinearity, from the last equation (48) one can retrieve (8) whenever the conditions (9) and

$$
2 n \eta_{l}+\xi_{l}+(2 n+1) \lambda_{l}=0 .
$$

stay valid. Consequently, in this scenario, it is also possible to retreive (11), and consequently (12). In view of this constraints the real portion (47) reduces to

$$
\begin{aligned}
& a \frac{\partial^{2} P_{l}}{\partial x^{2}}+b \frac{\partial^{2} P_{l}}{\partial x \partial t}+\{\kappa(\alpha+b \omega-a \kappa)-\omega\} P_{l} \\
& \quad+\left\{c_{l}+\kappa\left(\xi_{l}+\lambda_{l}\right)\right\} P_{l}^{2 n+1}=k_{l} P_{\bar{l}}
\end{aligned}
$$

As for Kerr nonlinearity, the last equation will be integrated implementing the ansatz approach in the next few subsections to examine four different forms of soliton solutions.

\subsubsection{Bright solitons}

To explore the details of bright soliton propagation on the system (45)-(46) we adopt the same starting hypothesis as for cubic nonlinearity, e.g. the one given by (17) jointly with the argument (18). The substitution of (17) into (50) leads to

$$
\begin{aligned}
& \left\{[\kappa(\alpha+b \omega-a \kappa)-\omega]+p_{l}^{2}(a-b \nu) B^{2}\right\} A_{l} \operatorname{sech}^{p_{l}} \tau \\
& \quad-p_{l}\left(1+p_{l}\right)(a-b \nu) A_{l} B^{2} \operatorname{sech}^{p_{l}+2} \tau \\
& \quad+\left[c_{l}+\kappa\left(\xi_{l}+\lambda_{l}\right)\right] A_{l}^{2 n+1} \operatorname{sech}^{(2 n+1) p_{l}} \tau \\
& \quad-k_{l} A_{\bar{l}} \operatorname{sech}^{p_{\bar{l}}} \tau=0
\end{aligned}
$$

The balance between nonlinearity and dispersion yield

$$
(2 n+1) p_{l}=p_{l}+2
$$

from which

$$
p_{l}=\frac{1}{n}
$$

for $l=1$ and $l=2$. Then, after the substitution of $(52)$ into (51), and setting the coefficients of the linearly independent functions $\operatorname{sech}^{j} \tau$ to zero for $j=\frac{1}{n}, \frac{2 n+1}{n}$ one get

$$
\nu=\frac{(n+1) a B^{2}-n^{2}\left\{c_{l}+\kappa\left(\lambda_{l}+\xi_{l}\right)\right\} A_{l}^{2 n}}{(n+1) b B^{2}}
$$

and

$$
\begin{gathered}
\omega=\left[(n+1) k_{l} A_{\bar{l}}-\left\{c_{l}+\kappa\left(\lambda_{l}+\xi_{l}\right)\right\} A_{l}^{2 n+1}\right. \\
\left.-(n+1) \kappa(\alpha-a \kappa) A_{l}\right] /\left\{2(b \kappa-1) A_{l}\right\}
\end{gathered}
$$

respectively, as long as $b \kappa \neq 1$ and $b B \neq 0$ hold. By comparing both alternative expressions for the soliton speed $\nu$ for $l=1,2$ in (53) a relation between the amplitudes takes the form

$$
\frac{A_{1}}{A_{2}}=\left[\frac{c_{2}+\kappa\left(\lambda_{2}+\xi_{2}\right)}{c_{1}+\kappa\left(\lambda_{1}+\xi_{1}\right)}\right]^{\frac{1}{2 n}}
$$

where $l=1,2$ and $\bar{l}=3-l$, subject to condition (24). In a similar manner, equating the two expressions for the soliton wave numbers from (54) for $l=1,2$ the connec- 
tion between the amplitudes of bright solitons in the two components is obtained as

$$
\begin{aligned}
& (n+1)\left(k_{1} A_{2}^{2}-k_{2} A_{1}^{2}\right)=A_{1} A_{2}\left\{\left[c_{1}+\kappa\left(\lambda_{1}+\xi_{1}\right)\right] A_{1}^{2 n}\right. \\
& \left.\quad-\left[c_{2}+\kappa\left(\lambda_{2}+\xi_{2}\right)\right] A_{2}^{2 n}\right\} .
\end{aligned}
$$

With the aid of (55), comparing (12) and (53) for either $l=1$ or $l=2$ yield

$$
A_{l}=\left[-\frac{(n+1)[a(1+b \kappa)-b(\alpha+b \omega)] B^{2}}{n^{2}(b \kappa-1)\left(c_{l}+\kappa\left(\lambda_{l}+\xi_{l}\right)\right.}\right]^{\frac{1}{2 n}}
$$

as long as the inequality (27) is satisfied. Thus, the bright soliton solution for the system describing dispersive directional couplers (45)-(46) with power law nonlinearity is given by

$$
\begin{gathered}
q(x, t)=A_{1} \operatorname{sech}^{\frac{1}{n}}[B(x-\nu t)] \mathrm{e}^{\mathrm{i}(-\kappa x+\omega t+\Theta)} \\
r(x, t)=A_{2} \operatorname{sech}^{\frac{1}{n}}[B(x-\nu t)] \mathrm{e}^{\mathrm{i}(-\kappa x+\omega t+\Theta)}
\end{gathered}
$$

The persistence of these bright soliton solution will be governed by the conditions discussed above.

\subsubsection{Dark solitons}

For the dark solitons the guess function to be taken is the same as in (31). Consequently the real component $(50)$ reduces to

$$
\begin{aligned}
& \left\{[\kappa(\alpha+b \omega-a \kappa)-\omega]-2 p_{l}^{2}(a-b \nu) B^{2}\right\} A_{l} \tanh ^{p_{l}} \tau \\
& \quad+p_{l}\left(p_{l}-1\right)(a-b \nu) A_{l} B^{2} \tanh ^{p_{l}-2} \tau \\
& \quad+p_{l}\left(p_{l}+1\right)(a-b \nu) A_{l} B^{2} \tanh ^{p_{l}+2} \tau \\
& \quad+\left[c_{l}+\kappa\left(\xi_{l}+\lambda_{l}\right)\right] A_{l}^{2 n+1} \tanh ^{(2 n+1) p_{l}} \tau \\
& \quad-k_{l} A_{\bar{l}} \tanh ^{p_{\bar{l}}} \tau=0 .
\end{aligned}
$$

In this case the balancing principle allow to retrieve the value of the parameter $p_{l}$ as in (52). However, the standalone element $\tanh ^{p_{l}-2} \tau$ leads to $p_{l}$ as the one given in (20) forcing $n=1$. Consequently the system (45)(46) reduces to (14)-(15), and the dark soliton solution will exist in nonlinear directional couplers whenever the power law nonlinearity collapses to Kerr law. Subsequently, the results for this subsection will be the same as of dark solitons for cubic nonlinearity (32)-(38) as well.

\subsubsection{Singular solitons (type-I)}

To investigate the first type of singular soliton solution of the system (45)-(46), the trial function (39) is again selected as a possible type-I pulse shape. The chosen guess reduces the real portion equation (50) into

$$
\begin{aligned}
& \{[ \\
& \left.\quad \kappa(\alpha+b \omega-a \kappa)-\omega]+p_{l}^{2}(a-b \nu) B^{2}\right\} A_{l} \operatorname{csch}^{p_{l}} \tau \\
& \quad+p_{l}\left(1+p_{l}\right)(a-b \nu) A_{l} B^{2} \operatorname{csch}^{p_{l}+2} \tau \\
& \quad+\left[c_{l}+\kappa\left(\xi_{l}+\lambda_{l}\right)\right] A_{l}^{2 n+1} \operatorname{csch}^{(2 n+1) p_{l}} \tau \\
& \quad-k_{l} A_{\bar{l}} \operatorname{csch}^{p_{\bar{l}}} \tau=0 .
\end{aligned}
$$

By making use of the appropriate balance between dispersion and nonlinear terms one get the value of $p_{l}$ as the one resulting in (52). Thus in (56), from the coefficients of the linearly independent functions $\operatorname{csch}^{j} \tau$ for $j=\frac{1}{n}$ and $j=\frac{2 n+1}{n}$, the soliton speed and wave numbers take the form

$$
\nu=\frac{(n+1) a B^{2}+n^{2}\left\{c_{l}+\kappa\left(\lambda_{l}+\xi_{l}\right)\right\} A_{l}^{2 n}}{(n+1) b B^{2}}
$$

and

$$
\begin{gathered}
\omega=\left[(n+1) k_{l} A_{\bar{l}}+\left[c_{l}+\kappa\left(\lambda_{l}+\xi_{l}\right)\right] A_{l}^{2 n+1}\right. \\
\left.-\{(n+1)(\alpha-a \kappa) \kappa\} A_{l}\right] / 2(b \kappa-1) A_{l} .
\end{gathered}
$$

Next, equating the expressions of $\nu$ for $l=1,2$ in $(57)$ leads to the quotient (55), while performing a similar procedure for $\omega$ in (58) yields the identity

$$
\begin{aligned}
& (n+1) k_{1} A_{2}^{2}+\left\{c_{1}+\kappa\left(\lambda_{1}+\xi_{1}\right)\right\} A_{1}^{2 n+1} A_{2} \\
& \quad=(n+1) k_{2} A_{1}^{2}+\left\{c_{2}+\kappa\left(\lambda_{2}+\xi_{2}\right)\right\} A_{1} A_{2}^{2 n+1} .
\end{aligned}
$$

For this kind of soliton and under power law nonlinearity, equating (12) and (57) either for $l=1$ or $l=2$ yield

$$
A_{l}=
$$

$$
\sqrt[1 / 2 n]{\frac{(n+1) B^{2}[a(1+b \kappa)-b(\alpha+b \omega)]\left[c_{\bar{l}}+\kappa\left(\lambda_{\bar{l}}+\xi_{\bar{l}}\right)\right]}{n^{2}(b \kappa-1)\left[\left(c_{l}+\kappa\left(\lambda_{l}+\xi_{l}\right)\left(c_{\bar{l}}+\kappa\left(\lambda_{\bar{l}}+\xi_{\bar{l}}\right)\right]\right.\right.}}
$$

after considering (55), and the inequality (37) holds. Therefore, the type-I singular soliton solution for the system (45)-(46) is

$$
\begin{gathered}
q(x, t)=A_{1} \operatorname{csch}^{1 / n}[B(x-\nu t)] \mathrm{e}^{\mathrm{i}(-\kappa x+\omega t+\Theta)} \\
r(x, t)=A_{2} \operatorname{csch}^{1 / n}[B(x-\nu t)] \mathrm{e}^{\mathrm{i}(-\kappa x+\omega t+\Theta)}
\end{gathered}
$$

where the parameters with corresponding constraints are summarized in (56)-(59).

\subsubsection{Singular solitons (type-II)}

Type-II singular soliton solution is the last kind of solitons to be considered for the system with power law nonlinearity (45)-(46). In order to solve the system under consideration, the substitution of the starting hypothesis given by (43) reduces (50) into

$$
\begin{aligned}
& \left\{[\kappa(\alpha+b \omega-a \kappa)-\omega]-2 p_{l}^{2}(a-b \nu) B^{2}\right\} A_{l} \operatorname{coth}^{p_{l}} \tau \\
& +p_{l}\left(p_{l}-1\right)(a-b \nu) A_{l} B^{2} \operatorname{coth}^{p_{l}-2} \tau \\
& +p_{l}\left(p_{l}+1\right)(a-b \nu) A_{l} B^{2} \operatorname{coth}^{p_{l}+2} \tau \\
& +\left[c_{l}+\kappa\left(\xi_{l}+\lambda_{l}\right)\right] A_{l}^{2 n+1} \operatorname{coth}^{(2 n+1) p_{l}} \tau \\
& -k_{l} A_{\bar{l}} \operatorname{coth}^{p_{\bar{l}}} \tau=0 \text {. }
\end{aligned}
$$

As expected, the balancing principle yield the same value of $p_{l}$ as in (52). However, the stand alone element $\operatorname{coth}^{p_{l}-2} \tau$ leads to $p_{l}=1$. Consequently, the system (45)-(46) reduces to (14)-(15), and the type-II singular soliton solution will exist in dispersive nonlinear directional couplers whenever the power law nonlinearity collapses to Kerr law, as as we found for dark solitons. As a consequence, the results for this subsection will be the same as of singular type-II solitons for cubic nonlinearity $(33)-(37)$. 


\section{Multiple-core couplers (coupling with nearest neighbors)}

The proposed model describing dispersive optical solitons in multiple-core couplers, while coupling with nearest neighbors, is given by

$$
\begin{array}{r}
\mathrm{i} q_{t}^{(l)}+\mathrm{i} \alpha_{l} q_{x}^{(l)}+a_{l} q_{x x}^{(l)}+b_{l} q_{x t}^{(l)}+F\left(\left|q^{(l)}\right|^{2}\right) q^{(l)} \\
+\mathrm{i} \Gamma_{l} q_{x x x}^{(l)}+\mathrm{i} \lambda_{l}\left(\left|q^{(l)}\right|^{2} q^{(l)}\right)_{x}+\mathrm{i} \xi_{l}\left|q^{(l)}\right|^{2} q_{x}^{(l)} \\
+\mathrm{i} \eta_{l}\left(\left|q^{(l)}\right|^{2}\right)_{x} q^{(l)}=\mathcal{K}\left[q^{(l-1)}-2 q^{(l)}+q^{(l+1)}\right]
\end{array}
$$

where $1 \leq l \leq N$. Equation (60) represents the general model for optical couplers where coupling with nearest neighbors is considered. For this model, $\mathcal{K}$ will be acting as the coupling coefficient. In order to address this model for the two forms of nonlinear media (Kerr and power Law), the initial hypothesis is taken to be

$$
q^{(l)}(x, t)=P_{l}(x, t) \mathrm{e}^{\mathrm{i} \phi(x, t)}
$$

where $P_{l}$ keeps the same amplitude meaning as before, while $\phi$ is the phase component and is again defined as in (5). By substituting the ansatz (61) into (60), the resulting expression is separated into the real and imaginary parts. The resulting imaginary portion is identical to (7), and as a direct consequence, one retrieve the soliton speed (8) jointly with solvability conditions (9) and (10) regardless any of the two nonlinearities to be taken into consideration. In addition, the real part takes the following updated form

$$
\begin{aligned}
& a_{l} \frac{\partial^{2} P_{l}}{\partial x^{2}}+b_{l} \frac{\partial^{2} P_{l}}{\partial x \partial t}+\left\{\kappa\left(\alpha_{l}+b \omega-a_{l} \kappa\right)-\omega\right\} P_{l} \\
& \quad+\kappa\left(\xi_{l}+\lambda_{l}\right) P_{l}^{3}+F\left(P_{l}^{2}\right) P_{l}=\mathcal{K}\left[P_{l-1}-2 P_{l}+P_{l+1}\right]
\end{aligned}
$$

in view of (9). These last equation will be analyzed for four different types of solitons in view of cubic and power law nonlinear forms.

\subsection{Kerr law nonlinearity}

In the case of Kerr law nonlinearity, the system (60) modifies to

$$
\begin{aligned}
& \mathrm{i} q_{t}^{(l)}+\mathrm{i} \alpha_{l} q_{x}^{(l)}+a_{l} q_{x x}^{(l)}+b_{l} q_{x t}^{(l)}+c_{l}\left|q^{(l)}\right|^{2} q^{(l)} \\
& \quad+\mathrm{i} \Gamma_{l} q_{x x x}^{(l)}+\mathrm{i} \lambda_{l}\left(\left|q^{(l)}\right|^{2} q^{(l)}\right)_{x}
\end{aligned}
$$

$+\mathrm{i} \xi_{l}\left|q^{(l)}\right|^{2} q_{x}^{(l)}+\mathrm{i} \eta_{l}\left(\left|q^{(l)}\right|^{2}\right)_{x} q^{(l)}=\mathcal{K}\left[q^{(l-1)}-2 q^{(l)}+q^{(l+1}\right.$

In view of the ansatz (61) considered herein, the real part equation $(62)$ is rewritten as

$$
\begin{aligned}
& a_{l} \frac{\partial^{2} P_{l}}{\partial x^{2}}+b_{l} \frac{\partial^{2} P_{l}}{\partial x \partial t}+\left\{\kappa\left(\alpha_{l}+b \omega-a \kappa\right)-\omega\right\} P_{l} \\
& \quad+\left\{c_{l}+\kappa\left(\xi_{l}+\lambda_{l}\right)\right\} P_{l}^{3}= \\
& \quad \mathcal{K}\left[P_{l-1}-2 P_{l}+P_{l+1}\right]
\end{aligned}
$$

The equation (64) will be further split into four subsections depending on the type of soliton that is being considered.

\subsubsection{Bright solitons}

When studying the dynamics of bright solitons in system (64), the starting hypothesis to be taken is given by

$$
P_{l}=A_{l} \operatorname{sech}^{p} \tau
$$

where the argument is defined by

$$
\tau=B(x-\nu t),
$$

$p$ is a parameter to be determined, while $A_{l}$ and $B$ dictates the soliton amplitude and inverse width respectively. The substitution of the hypothesis (65) in (64) convert the last into

$$
\begin{aligned}
& \left\{\left[\kappa\left(\alpha_{l}+b_{l} \omega-a_{l} \kappa\right)-\omega\right]+p^{2}\left(a_{l}-b_{l} \nu\right) B^{2}\right\} A_{l} \operatorname{sech}^{p} \tau \\
& \quad-p(1+p)\left(a_{l}-b_{l} \nu\right) A_{l} B^{2} \operatorname{sech}^{p+2} \tau \\
& \quad+\left[c_{l}+\kappa\left(\xi_{l}+\lambda_{l}\right)\right] A_{l}^{3} \operatorname{sech}^{3 p} \tau \\
& \quad-\mathcal{K}\left(A_{l-1}-2 A_{l}+A_{l+1}\right) \operatorname{sech}^{p} \tau=0
\end{aligned}
$$

The expected balancing principle gives

$$
p=1 \text {. }
$$

Then, after setting the coefficients of the linearly independent functions to zero yields the waves number

$\omega=$

$$
\frac{2 \mathcal{K}\left(A_{l-1}-2 A_{l}+A_{l+1}\right)-\Gamma_{l} A_{l}^{3}-2 \kappa\left(\alpha_{l}-a_{l} \kappa\right) A_{l}}{2\left(b_{l} \kappa-1\right) A_{l}},
$$

and the soliton speed

$$
\nu=\frac{2 a_{l} B^{2}-\Gamma_{l} A_{l}^{2}}{2 b_{l} B^{2}},
$$

where $\Gamma_{l}=c_{l}+\kappa\left(\lambda_{l}+\xi_{l}\right)$. Next, equating the two expressions of the soliton speed $\nu,(8)$ and (68) results in

$$
B=
$$

$$
\sqrt{\frac{\left(b_{l} \kappa-1\right)^{2} \Gamma_{l} A_{l}^{3}}{2 a_{l} A_{l}+b_{l}\left\{2 b_{l} \mathcal{K}\left(A_{l-1}-2 A_{l}+A_{l+1}\right)-2 \alpha_{l} A_{l}-b_{l} \Gamma_{l} A_{l}^{3}\right\}}}
$$

in view of (67), as long as

$$
\begin{aligned}
& \Gamma_{l} A_{l}^{3}\left[2 a_{l} A_{l}+b_{l}\left\{2 b_{l} \mathcal{K}\left(A_{l-1}-2 A_{l}+A_{l+1}\right)\right.\right. \\
& \left.\left.\quad-2 \alpha_{l} A_{l}-b_{l} \Gamma_{l} A_{l}^{3}\right\}\right]>0
\end{aligned}
$$

Hence, the bright soliton solution to the system (63) is

$$
q^{(l)}(x, t)=A_{l} \operatorname{sech}[B(x-\nu t)] \mathrm{e}^{\mathrm{i}(-\kappa x+\omega t+\Theta)}
$$

where the amplitude and the inverse width are related iin (69) and the soliton speed can be either (8) or (68), as long as the corresponding conditions hold.

\subsubsection{Dark solitons}

To study dark solitons solution on the system (63), we assume a solution of the form

$$
P_{l}=A_{l} \tanh ^{p} \tau \text {. }
$$

The substitution of (70) into (64) leads to

$$
\begin{aligned}
& \left\{\left[\kappa\left(\alpha_{l}+b_{l} \omega-a_{l} \kappa\right)-\omega\right]\right. \\
& \left.\quad-2 p^{2}\left(a_{l}-b_{l} \nu\right) B^{2}\right\} A_{l} \tanh ^{p} \tau \\
& \quad+p(p-1)\left(a_{l}-b_{l} \nu\right) A_{l} B^{2} \tanh ^{p-2} \tau
\end{aligned}
$$




$$
\begin{aligned}
& +p(p+1)\left(a_{l}-b_{l} \nu\right) A_{l} B^{2} \tanh ^{p+2} \tau \\
& +\left[c_{l}+\kappa\left(\xi_{l}+\lambda_{l}\right)\right] A_{l}^{3} \tanh ^{3 p} \tau \\
& -\mathcal{K}\left(A_{l-1}-2 A_{l}+A_{l+1}\right) \tanh ^{p} \tau=0
\end{aligned}
$$

The balancing principle, as well as the standalone linearly independent function $\tanh ^{p_{l}-2} \tau$ leads one to retrieve the value of the parameter $p$ given in (66). Thus, setting to zero the coefficients of the linearly independent functions $\tanh ^{j} \tau$ for $j=1,3$ leads to the speed of the soliton

$$
\nu=\frac{2 a_{l} B^{2}+\left[c_{l}+\kappa\left(\lambda_{l}+\xi_{l}\right)\right] A_{l}^{2}}{2 b_{l} B^{2}}
$$

and the wave number

$$
\begin{aligned}
\omega & =\left[\mathcal{K}\left(A_{l-1}-2 A_{l}+A_{l+1}\right)-\left\{c_{l}+\kappa\left(\lambda_{l}+\xi_{l}\right)\right\} A_{l}^{3}\right. \\
& \left.-\kappa\left(\alpha_{l}-a_{l} \kappa\right) A_{l}\right] /\left(b_{l} \kappa-1\right) A_{l} .
\end{aligned}
$$

By comparing (8) and (71), the soliton amplitude and inverse width are associated through

$$
B=
$$

$\sqrt{\frac{\left(b_{l} \kappa-1\right)^{2} \Gamma_{l} A_{l}^{3}}{2 a_{l} A_{l}+2 b_{l}\left\{b_{l} \mathcal{K}\left(A_{l-1}-2 A_{l}+A_{l+1}\right)-\alpha_{l} A_{l}-b_{l} \Gamma_{l} A_{l}^{3}\right\}}}$

after considering (72), whenever the corresponding radicand is positive. Therefore, the dark soliton solution to the system $(63)$ is

$$
q^{(l)}(x, t)=A_{l} \tanh [B(x-\nu t)] \mathrm{e}^{\mathrm{i}(-\kappa x+\omega t+\Theta)}
$$

where the parameters along with corresponding constraints were discussed above.

\subsubsection{Singular solitons (type-I)}

For type-I singular soliton the ansatz is of the form

$$
P_{l}=A_{l} \operatorname{csch}^{p} \tau \text {. }
$$

Substituting (75) into (64) generates

$$
\begin{aligned}
& \left\{\left[\kappa\left(\alpha_{l}+b_{l} \omega-a_{l} \kappa\right)-\omega\right]+p^{2}\left(a_{l}-b_{l} \nu\right) B^{2}\right\} A_{l} \operatorname{csch}^{p} \tau \\
& \quad+p(1+p)\left(a_{l}-b_{l} \nu\right) A_{l} B^{2} \operatorname{csch}^{p+2} \tau \\
& \quad+\left[c_{l}+\kappa\left(\xi_{l}+\lambda_{l}\right)\right] A_{l}^{3} \operatorname{csch}^{3 p} \tau \\
& \quad-\mathcal{K}\left(A_{l-1}-2 A_{l}+A_{l+1}\right) \operatorname{csch}^{p} \tau=0 .
\end{aligned}
$$

after simplification. Once again the balance between nonlinearity and dispersion leads to the value of $p$ obtained in (66). After substituting $p=1$ in the expression (76), and setting the coefficients of the linearly independent functions $\operatorname{csch}^{j} \tau$ for $j=1,3$ to zero allow to retrieve the same soliton speed as for dark soliton (71), but the wave number becomes:

$$
\begin{aligned}
\omega= & {\left[2 \mathcal{K}\left(A_{l-1}-2 A_{l}+A_{l+1}\right)+\Gamma_{l} A_{l}^{3}-2 \kappa\left(\alpha_{l}-a_{l} \kappa\right]\right.} \\
& / 2\left(b_{l} \kappa-1\right) A_{l} .
\end{aligned}
$$

where $\Gamma_{l}=c_{l}+\kappa\left(\lambda_{l}+\xi_{l}\right)$. Next, comparing the speeds (8) and (71), and using (77) leads to

$$
B=
$$

$\sqrt{-\frac{\left(b_{l} \kappa-1\right)^{2} \Gamma_{l} A_{l}^{3}}{2 a_{l} A_{l}+b_{l}\left\{2 b_{l} \mathcal{K}\left(A_{l-1}-2 A_{l}+A_{l+1}\right)-2 \alpha_{l} A_{l}+b_{l} \Gamma_{l} A_{l}^{3}\right\}}}$

provided

$$
\begin{aligned}
& \Gamma_{l} A_{l}^{3}\left[2 a_{l} A_{l}+b_{l}\left\{2 b_{l} \mathcal{K}\left(A_{l-1}-2 A_{l}+A_{l+1}\right)\right.\right. \\
& \left.\left.\quad-2 \alpha_{l} A_{l}+b_{l} \Gamma_{l} A_{l}^{3}\right\}\right]<0
\end{aligned}
$$

Thus the type-I singular soliton solution to the system $(63)$ is

$$
q^{(l)}(x, t)=A_{l} \operatorname{csch}[B(x-\nu t)] \mathrm{e}^{\mathrm{i}(-\kappa x+\omega t+\Theta)}
$$

where the parameters along with corresponding constraints are discussed as above.

\subsubsection{Singular solitons (type-II)}

For the second type of singular soliton solution of the system (63) we assume a solution of the following form,

$$
P_{l}=A_{l} \operatorname{coth}^{p} \tau
$$

and substitute the same in the real part (64) to obtain

$$
\begin{aligned}
& \{[ \\
& \left.\quad \kappa\left(\alpha_{l}+b_{l} \omega-a_{l} \kappa\right)-\omega\right] \\
& \left.\quad-2 p^{2}\left(a_{l}-b_{l} \nu\right) B^{2}\right\} A_{l} \operatorname{coth}^{p} \tau \\
& \quad+p(p-1)\left(a_{l}-b_{l} \nu\right) A_{l} B^{2} \operatorname{coth}^{p-2} \tau \\
& \quad+p(p+1)\left(a_{l}-b_{l} \nu\right) A_{l} B^{2} \operatorname{coth}^{p+2} \tau \\
& \quad+\left[c_{l}+\kappa\left(\xi_{l}+\lambda_{l}\right)\right] A_{l}^{3} \operatorname{coth}^{3 p} \tau \\
& \quad-\mathcal{K}\left(A_{l-1}-2 A_{l}+A_{l+1}\right) \operatorname{coth}^{p} \tau=0 .
\end{aligned}
$$

Notice that the coefficient of the stand alone element $\operatorname{coth}^{p_{l}-2} \tau$ yields the same value of the parameter $p$ as in (66). The balancing principle yields unity as well. Thus, the substitution of $p=1$ into (79) leads to the same results as for dark solitons (71)-(73) together with corresponding conditions. Therefore, the type-II singular soliton solution for the nonlinear system (63) has the form

$$
q^{(l)}(x, t)=A_{l} \operatorname{coth}[B(x-\nu t)] \mathrm{e}^{\mathrm{i}(-\kappa x+\omega t+\Theta)}
$$

with parameters along with corresponding constraints are the same as for dark soliton in the case of multiple-core couplers when coupling with the nearest neighbors with Kerr law nonlinearity.

\subsection{Power law nonlinearity}

When considering power law nonlinearity the system $(60)$ is rewritten as

$$
\begin{gathered}
\mathrm{i} q_{t}^{(l)}+\mathrm{i} \alpha_{l} q_{x}^{(l)}+a_{l} q_{x x}^{(l)}+b_{l} q_{x t}^{(l)}+c_{l}\left|q^{(l)}\right|^{2 n} q^{(l)} \\
+\mathrm{i} \Gamma_{l} q_{x x x}^{(l)}+\mathrm{i} \lambda_{l}\left(\left|q^{(l)}\right|^{2 n} q^{(l)}\right)_{x}+\mathrm{i} \xi_{l}\left|q^{(l)}\right|^{2 n} q_{x}^{(l)} \\
+\mathrm{i} \eta_{l}\left(\left|q^{(l)}\right|^{2 n}\right)_{x} q^{(l)}=\mathcal{K}\left[q^{(l-1)}-2 q^{(l)}+q^{(l+1)}\right]
\end{gathered}
$$

In this context, the substitution of the starting hypothesis (61) into (80) leads to retrieve the same imaginary part we got on (48), thus one can retrieve (8) whenever the conditions (9) and (49) hold. Consequently, the resulting real part can be rewritten as 


$$
\begin{aligned}
& a_{l} \frac{\partial^{2} P_{l}}{\partial x^{2}}+b_{l} \frac{\partial^{2} P_{l}}{\partial x \partial t}+\left\{\kappa\left(\alpha_{l}+b_{l} \omega-a_{l} \kappa\right)-\omega\right\} P_{l} \\
& \quad+\left\{c_{l}+\kappa\left(\xi_{l}+\lambda_{l}\right)\right\} P_{l}^{2 n+1}= \\
& \quad \mathcal{K}\left[P_{l-1}-2 P_{l}+P_{l+1}\right] .
\end{aligned}
$$

The equation (81) will be further split into four subsections depending on the type of soliton that is being considered.

\subsubsection{Bright solitons}

To investigate the bright soliton propagation on the system (80) we adopt the ansatz (65), and substitute the same into (81) to obtain

$$
\begin{aligned}
& \{[ \\
& \left.\left.\quad \kappa\left(\alpha_{l}+b_{l} \omega-a_{l} \kappa\right)-\omega\right]+p^{2}\left(a_{l}-b_{l} \nu\right) B^{2}\right\} A_{l} \operatorname{sech}^{p} \tau \\
& \quad-p(1+p)\left(a_{l}-b_{l} \nu\right) A_{l} B^{2} \operatorname{sech}^{p+2} \tau \\
& \quad+\left[c_{l}+\kappa\left(\xi_{l}+\lambda_{l}\right)\right] A_{l}^{2 n+1} \operatorname{sech}^{(2 n+1) p} \tau \\
& \quad-\mathcal{K}\left[A_{l-1}-2 A_{l}+A_{l+1}\right] \operatorname{sech}^{p} \tau=0
\end{aligned}
$$

By comparing dispersion and nonlinearity in the last one leads to

$$
(2 n+1) p=p+2
$$

from which

$$
p=\frac{1}{n} \text {. }
$$

The substitution of (83) into (82), and setting the coefficients of the linearly independent functions $\operatorname{sech}^{j} \tau$ to zero generates the soliton speed

$$
\nu=\frac{(n+1) a_{l} B^{2}-n^{2} \Gamma_{l} A_{l}^{2 n}}{(n+1) b_{l} B^{2}},
$$

where by convenience we have defined $\Gamma_{l}=c_{l}+\kappa\left(\lambda_{l}+\xi_{l}\right)$, and the wave number

$$
\begin{gathered}
\omega=\left[(n+1) \mathcal{K}\left(A_{l-1}-2 A_{l}+A_{l+1}\right)-\Gamma_{l} A_{l}^{2 n+1}\right. \\
\left.-(n+1) \kappa\left(\alpha_{l}-a_{l} \kappa\right) A_{l}\right] /(n+1)\left(b_{l} \kappa-1\right) A_{l},
\end{gathered}
$$

whenever $b \kappa \neq 1$ and $b B \neq 0$. Then, after comparing the two possible expressions of the soliton speed $\nu,(8)$ and (84) one obtain

$$
B=
$$

$$
\sqrt{\frac{n^{2}\left(b_{l} \kappa-1\right)^{2} \Gamma_{l} A_{l}^{2 n+1}}{(n+1)\left[\left(a_{l}-b_{l} \alpha_{l}\right) A_{l}+b_{l}^{2} \mathcal{K}\left(A_{l-1}-2 A_{l}+A_{l+1}\right)\right]-b_{l}^{2} \Gamma_{l} A_{l}^{2 n+1}}}
$$

Therefore the bright 1 -soliton solution is given by

$$
q^{(l)}(x, t)=A_{l} \operatorname{sech}^{\frac{1}{n}}[B(x-\nu t)] \mathrm{e}^{\mathrm{i}(-\kappa x+\omega t+\Theta)}
$$

where the association between the amplitudes and the inverse width is depicted in (86), the soliton speed can be either (8) or (84), and the wave number is defined in (85). The corresponding constraints have to be satisfies in order for the soliton to propagate within the directional couplers described by the system (80).

\subsubsection{Dark solitons}

For dark soliton the starting hypothesis is as in (70), and its substitution in (81) prompt to

$\left\{\left[\kappa\left(\alpha_{l}+b_{l} \omega-a_{l} \kappa\right)-\omega\right]-2 p^{2}\left(a_{l}-b_{l} \nu\right) B^{2}\right\} A_{l} \tanh ^{p} \tau$

$$
\begin{aligned}
& +p(p-1)\left(a_{l}-b_{l} \nu\right) A_{l} B^{2} \tanh ^{p-2} \tau \\
& +p(p+1)\left(a_{l}-b_{l} \nu\right) A_{l} B^{2} \tanh ^{p+2} \tau \\
& +\left[c_{l}+\kappa\left(\xi_{l}+\lambda_{l}\right)\right] A_{l}^{2 n+1} \tanh ^{(2 n+1) p} \tau \\
& -\mathcal{K}\left(A_{l-1}-2 A_{l}+A_{l+1}\right) \tanh ^{p} \tau=0 .
\end{aligned}
$$

The balance between dispersion and nonlinearity leads to the value of the parameter $p$ as in (83). On the other hand, the standalone element $\tanh ^{p-2} \tau$ leads to $p=1$, therefore $n=1$. As a direct consequence, the system with power law nonlinearity (80) collapses to the one with Kerr law (63), whose results were discussed in (71)-(73).

\subsubsection{Singular solitons (type-I)}

For singular type-I soliton solution we adopt and substitute the waveform (75) into (81) giving

$$
\begin{aligned}
& \left\{\left[\kappa\left(\alpha_{l}+b_{l} \omega-a_{l} \kappa\right)-\omega\right]+p^{2}\left(a_{l}-b_{l} \nu\right) B^{2}\right\} A_{l} \operatorname{csch}^{p} \tau \\
& \quad+p(1+p)\left(a_{l}-b_{l} \nu\right) A_{l} B^{2} \operatorname{csch}^{p+2} \tau \\
& \quad+\left[c_{l}+\kappa\left(\xi_{l}+\lambda_{l}\right)\right] A_{l}^{2 n+1} \operatorname{csch}^{(2 n+1) p} \tau \\
& \quad-\mathcal{K}\left(A_{l-1}-2 A_{l}+A_{l+1}\right) \operatorname{csch}^{p} \tau=0 .
\end{aligned}
$$

Balancing principle leads the same value of $p$ as in (83). Thus, from the linearly independent function, the speed and wave number are

$$
\nu=\frac{(n+1) a_{l} B^{2}+n^{2} \Gamma_{l} A_{l}^{2 n}}{(n+1) b_{l} B^{2}}
$$

and

$$
\begin{gathered}
\omega=\left[(n+1) \mathcal{K}\left(A_{l-1}-2 A_{l}+A_{l+1}\right)+\Gamma_{l} A_{l}^{2 n+1}\right. \\
-(n+1) \kappa(\alpha-a \kappa)] /(n+1)\left(b_{l} \kappa-1\right) A_{l} .
\end{gathered}
$$

respectively, where we have define by convenience $\Gamma_{l}=$ $c_{l}+\kappa\left(\xi_{l}+\lambda_{l}\right)$. A direct comparison of the soliton speeds (8) and (87) leads to

$$
\sqrt{\frac{B=}{(n+1)\left[\left(a_{l}-b_{l} \alpha_{l}\right) A_{l}+b_{l}^{2} \mathcal{K}\left(A_{l-1}-2 A_{l}+A_{l+1}\right)\right]+b_{l}^{2} \Gamma_{l} A_{l}^{2 n+1}}}
$$

subject to

$$
\begin{aligned}
& \Gamma_{l} A_{l}^{2 n+1} \times\left\{( n + 1 ) \left[\left(a_{l}-b_{l} \alpha_{l}\right) A_{l}\right.\right. \\
& \left.\left.\quad+b_{l}^{2} \mathcal{K}\left(A_{l-1}-2 A_{l}+A_{l+1}\right)\right]+b_{l}^{2} \Gamma_{l} A_{l}^{2 n+1}\right\}>0
\end{aligned}
$$

Therefore the singular type-I soliton solution to (80) is

$$
q^{(l)}(x, t)=A_{l} \operatorname{csch}^{\frac{1}{n}}[B(x-\nu t)] \mathrm{e}^{\mathrm{i}(-\kappa x+\omega t+\Theta)}
$$

where the amplitude, inverse width, speed, and wave number are presented above together with corresponding solvability conditions.

\subsubsection{Singular solitons (type-II)}

For power law nonlinearity, the last waveform to be studied in multiple-core couplers when coupling with nearest neighbors is the singular type-II, for which the starting hypothesis to be taken is the same as for the case of cubic nonlinearity, (70). Then, the waveform (70) reduces $(81)$ to 


$$
\begin{aligned}
& \left\{\left[\kappa\left(\alpha_{l}+b_{l} \omega-a_{l} \kappa\right)-\omega\right]-2 p^{2}\left(a_{l}-b_{l} \nu\right) B^{2}\right\} A_{l} \operatorname{coth}^{p} \tau \\
& \quad+p(p-1)\left(a_{l}-b_{l} \nu\right) A_{l} B^{2} \operatorname{coth}^{p-2} \tau \\
& \quad+p(p+1)\left(a_{l}-b_{l} \nu\right) A_{l} B^{2} \operatorname{coth}^{p+2} \tau \\
& \quad+\left[c_{l}+\kappa\left(\xi_{l}+\lambda_{l}\right)\right] A_{l}^{2 n+1} \operatorname{coth}^{(2 n+1) p} \tau \\
& \quad-\mathcal{K}\left(A_{l-1}-2 A_{l}+A_{l+1}\right) \operatorname{coth}^{p} \tau=0 .
\end{aligned}
$$

By balancing principle the parameter $p$ takes the same value as in (83), but the standalone element $\operatorname{coth}^{p-2} \tau$ leads to $p=1$. Thus, equating both values of $p$ makes $n=1$. Consequently, for the type- 2 singular soliton solution, the system with power law nonlinearity (80) reduces to the system with Kerr nonlinearity (63), whose results were discussed through (71)-(73) together with the corresponding constraints. Finally, the type-II singular soliton solution for the nonlinear system (63) has the form

$$
q^{(l)}(x, t)=A_{l} \operatorname{coth}[B(x-\nu t)] \mathrm{e}^{\mathrm{i}(-\kappa x+\omega t+\Theta)}
$$

where parameters along with corresponding constraints are the same as for dark soliton in the case of multiplecore couplers when coupling with the nearest neighbors with Kerr law nonlinearity.

\section{Multiple-core couplers (coupling with all neighbors)}

The governing system for multiple core couplers, where the coupling is with all neighbors, is

$$
\begin{aligned}
& \mathrm{i} q_{t}^{(l)}+\mathrm{i} \alpha_{l} q_{x}^{(l)}+a_{l} q_{x x}^{(l)}+b_{l} q_{x t}^{(l)}+F\left(\left|q^{(l)}\right|^{2}\right) q^{(l)} \\
& \quad+\mathrm{i} \Gamma_{l} q_{x x x}^{(l)}+\mathrm{i} \lambda_{l}\left(\left|q^{(l)}\right|^{2} q^{(l)}\right)_{x}+\mathrm{i} \xi_{l}\left|q^{(l)}\right|^{2} q_{x}^{(l)} \\
& \quad+\mathrm{i} \eta_{l}\left(\left|q^{(l)}\right|^{2}\right)_{x} q^{(l)}=\sum_{n=1}^{N} \delta_{l n} q^{(n)}
\end{aligned}
$$

where $1 \leq l \leq N$ and $\delta_{l n}$ represents the coupling coefficient with all neighbors. The initial hypothesis is taken to be the same as given by (61). Thus, the substitution of (61) into (88) allow to split into imaginary and real parts. The imaginary part is the same as (7), therefore one retrieve the soliton speed (8) subject to constraints (9) and (10) regardless any of the two nonlinearities (Kerr and power law) to be taken into consideration throughout the rest of the manuscript. Meanwhile, the real part takes the form

$$
\begin{gathered}
a_{l} \frac{\partial^{2} P_{l}}{\partial x^{2}}+b_{l} \frac{\partial^{2} P_{l}}{\partial x \partial t}+\left[\kappa\left(\alpha_{l}+b_{l} \omega-a_{l} \kappa\right)-\omega\right] P_{l} \\
+\kappa\left(\xi_{l}+\lambda_{l}\right) P_{l}^{3}+F\left(P_{l}^{2}\right) P_{l}=\sum_{n=1}^{N} \delta_{l n} P_{n} .
\end{gathered}
$$

For the remaining of this work, the last expression will be studied in view of both nonlinearities as follow.

\subsection{Kerr law nonlinearity}

If we first take into consideration the Kerr nonlinearity, the system (88) can be rewritten as

$$
\mathrm{i} q_{t}^{(l)}+\mathrm{i} \alpha_{l} q_{x}^{(l)}+a_{l} q_{x x}^{(l)}+b_{l} q_{x t}^{(l)}+c_{l}\left|q^{(l)}\right|^{2} q^{(l)}
$$

$$
\begin{aligned}
& +\mathrm{i} \Gamma_{l} q_{x x x}^{(l)}+\mathrm{i} \lambda_{l}\left(\left|q^{(l)}\right|^{2} q^{(l)}\right)_{x}+\mathrm{i} \xi_{l}\left|q^{(l)}\right|^{2} q_{x}^{(l)} \\
& +\mathrm{i} \eta_{l}\left(\left|q^{(l)}\right|^{2}\right)_{x} q^{(l)}=\sum_{n=1}^{N} \delta_{l n} q^{(n)} .
\end{aligned}
$$

The substitution of the starting hypothesis (61) into (89) generates the same imaginary part we got on (48), and as a direct consequence, one can retrieve (8) whenever the conditions (9) and (10) are satisfied. In this context, the resulting real part can be rewritten as

$$
\begin{aligned}
& a_{l} \frac{\partial^{2} P_{l}}{\partial x^{2}}+b_{l} \frac{\partial^{2} P_{l}}{\partial x \partial t}+\left\{\kappa\left(\alpha_{l}+b_{l} \omega-a_{l} \kappa\right)-\omega\right\} P_{l} \\
& \quad+\left\{c_{l}+\kappa\left(\xi_{l}+\lambda_{l}\right)\right\} P_{l}^{3}=\sum_{n=1}^{N} \delta_{l n} P_{n} .
\end{aligned}
$$

The equation (90) will be further split into four subsections depending on the type of soliton that is being considered.

\subsubsection{Bright solitons}

For bright solitons, the hypothesis given by (65) simplifies the real part equation (90) to

$$
\begin{aligned}
& \left\{\left[\kappa\left(\alpha_{l}+b_{l} \omega-a_{l} \kappa\right)-\omega\right]+p^{2}\left(a_{l}-b_{l} \nu\right) B^{2}\right\} A_{l} \operatorname{sech}^{p} \tau \\
& \quad-p(1+p)\left(a_{l}-b_{l} \nu\right) A_{l} B^{2} \operatorname{sech}^{p+2} \tau \\
& \quad+\left[c_{l}+\kappa\left(\xi_{l}+\lambda_{l}\right)\right] A_{l}^{3} \operatorname{sech}^{3 p} \tau=\operatorname{sech}^{p} \tau \sum_{n=1}^{N} \delta_{l n} A_{n}(91)
\end{aligned}
$$

where the balancing principle leads to $p=1$. Inserting such value of $p$ in (91), and setting the coefficients of the linearly independent functions $\operatorname{sech}^{j} \tau, j=1,3$ to zero leads to the soliton wave number

$$
\omega=\frac{-\Gamma_{l} A_{l}^{3}-2 \kappa\left(\alpha_{l}-a_{l} \kappa\right) A_{l}+2 \sum_{n=1}^{N} \delta_{l n} A_{n}}{2\left(b_{l} \kappa-1\right) A_{l}},
$$

where $\Gamma_{l}=c_{l}+\kappa\left(\lambda_{l}+\xi_{l}\right)$ while the speed (68) is retrieved, which after comparing with its equivalent (8), one get

$$
A_{l} \sqrt{\frac{\left(b_{l} \kappa-1\right)^{2} \Gamma_{l} A_{l}}{2 a_{l} A_{l}-b_{l}\left\{2 \alpha_{l} A_{l}+b_{l} \Gamma_{l} A_{l}^{3}-2 b_{l} \sum_{n=1}^{N} \delta_{l n} A_{n}\right\}}}
$$

in the context of (92), in addition to the constraints

$\Gamma_{l} A_{l}\left[2 a_{l} A_{l}-b_{l}\left\{2 \alpha_{l} A_{l}+b_{l} \Gamma_{l} A_{l}^{3}-2 b_{l} \sum_{n=1}^{N} \delta_{l n} A_{n}\right\}\right]>0$ and

$$
\left(b_{l} \kappa-1\right) A_{l} \neq 0 .
$$

Thus, we can deduce that the bright soliton solution to the system (89) is

$$
q^{(l)}(x, t)=A_{l} \operatorname{sech}[B(x-\nu t)] \mathrm{e}^{\mathrm{i}(-\kappa x+\omega t+\Theta)}
$$

where the parameters with corresponding solvability conditions that secure the persistence of such soliton are discussed above. 


\subsubsection{Dark solitons}

To explore dark soliton solution of the nonlinear system (89) we start by assuming a solution of the form (70). The substitution of such guess into (90) transform the last into

$$
\begin{aligned}
& \left\{\left[\kappa\left(\alpha_{l}+b_{l} \omega-a_{l} \kappa\right)-\omega\right]-2 p^{2}\left(a_{l}-b_{l} \nu\right) B^{2}\right\} A_{l} \tanh ^{p} \tau \\
& \quad+p(p-1)\left(a_{l}-b_{l} \nu\right) A_{l} B^{2} \tanh ^{p-2} \tau \\
& \quad+p(p+1)\left(a_{l}-b_{l} \nu\right) A_{l} B^{2} \tanh ^{p+2} \tau \\
& \quad+\left[c_{l}+\kappa\left(\xi_{l}+\lambda_{l}\right)\right] A_{l}^{3} \tanh ^{3 p} \tau= \\
& \quad \tanh ^{p} \tau \sum_{n=1}^{N} \delta_{l n} A_{n}
\end{aligned}
$$

The standalone linearly independent function $\tanh ^{p_{l}-2} \tau$ leads one to retrieve the value $p=1$, while the balancing principle yields the same. Setting to zero the coefficients of the linearly independent functions $\tanh ^{j} \tau$ for $j=1,3$ leads to the speed of the soliton (71), and the wave number

$$
\omega=\frac{-\Gamma_{l} A_{l}^{3}-\kappa\left(\alpha_{l}-a_{l} \kappa\right) A_{l}+\sum_{n=1}^{N} \delta_{l n} A_{n}}{\left(b_{l} \kappa-1\right) A_{l}} .
$$

with $\Gamma_{l}=c_{l}+\kappa\left(\lambda_{l}+\xi_{l}\right)$. As before, if we compare both possible expressions for the soliton speed (8) and (71), one can define the inverse width in terms of the amplitude by

$$
B=\sqrt{\frac{\left(b_{l} \kappa-1\right)^{2} \Gamma_{l} A_{l}^{3}}{2 a_{l} A_{l}-2 b_{l}\left\{\alpha_{l} A_{l}+b_{l} \Gamma_{l} A_{l}^{3}-b_{l} \sum_{n=1}^{N} \delta_{l n} A_{n}\right\}}}
$$

by virtue of (95), whenever the corresponding radicand stay positive, e.g. (93) and

$$
\begin{gathered}
\Gamma_{l} A_{l}^{3}\left[a_{l} A_{l}-b_{l}\left\{\alpha_{l} A_{l}+b_{l} \Gamma_{l} A_{l}^{3}-b_{l} \sum_{n=1}^{N} \delta_{l n} A_{n}\right\}\right] \\
\quad>0
\end{gathered}
$$

stay valid. Finally, the dark soliton solution to the system (89) is

$$
q^{(l)}(x, t)=A_{l} \tanh [B(x-\nu t)] \mathrm{e}^{\mathrm{i}(-\kappa x+\omega t+\Theta)}
$$

where the definition of each of the parameters and corresponding constraints have been discussed above.

\subsubsection{Singular solitons (type-I)}

For the first type of singular soliton in multiple core couplers with cubic nonlinearity, the starting hypothesis (75) simplifies the real part (90) to

$$
\begin{aligned}
& \left\{\left[\kappa\left(\alpha_{l}+b_{l} \omega-a_{l} \kappa\right)-\omega\right]+p^{2}\left(a_{l}-b_{l} \nu\right) B^{2}\right\} A_{l} \operatorname{csch}^{p} \tau \\
& \quad+p(1+p)\left(a_{l}-b_{l} \nu\right) A_{l} B^{2} \operatorname{csch}^{p+2} \tau \\
& \quad+\left[c_{l}+\kappa\left(\xi_{l}+\lambda_{l}\right)\right] A_{l}^{3} \operatorname{csch}^{3 p} \tau=\operatorname{csch}^{p} \tau \sum_{n=1}^{N} \delta_{l n} A_{n} .
\end{aligned}
$$

The balance between nonlinearity and dispersion yields again $p=1$. After substituting the resulting value of $p$ in the expression (97), and then setting the coefficients of the linearly independent functions $\operatorname{csch}^{j} \tau$ for $j=1,3$ to zero allow to retrieve the same expression for the soliton speed as for dark soliton (71), while an expression for for the wave number is,

$$
\omega=\frac{\Gamma_{l} A_{l}^{3}-2 \kappa\left(\alpha_{l}-a_{l} \kappa\right)+2 \sum_{n=1}^{N} \delta_{l n} A_{n}}{2\left(b_{l} \kappa-1\right) A_{l}} .
$$

with $\quad \Gamma_{l}=c_{l}+\kappa\left(\lambda_{l}+\xi_{l}\right)$. Next, comparing both speeds (8) and (71), and taking into consideration (98), the inverse width can be written in terms of the amplitude as

$$
B=
$$

$$
\sqrt{-\frac{\left(b_{l} \kappa-1\right)^{2} \Gamma_{l} A_{l}^{3}}{2 a_{l} A_{l}-b_{l}\left\{2 \alpha_{l} A_{l}-b_{l} \Gamma_{l} A_{l}^{3}-2 b_{l} \sum_{n=1}^{N} \delta_{l n} A_{n}\right\}}}
$$

as long as

$\Gamma_{l} A_{l}^{3}\left[2 a_{l} A_{l}-b_{l}\left\{2 \alpha_{l} A_{l}-b_{l} \Gamma_{l} A_{l}^{3}-2 b_{l} \sum_{n=1}^{N} \delta_{l n} A_{n}\right\}\right]<0$.

Thus, we can conclude that the type-I singular soliton solution to the system (89) is

$$
q^{(l)}(x, t)=A_{l} \operatorname{csch}[B(x-\nu t)] \mathrm{e}^{\mathrm{i}(-\kappa x+\omega t+\Theta)}
$$

where the speed can be consider as either (8) or (71), the wave number (98), and the relation between the inverse width and the amplitude by (99), all of these in view of the corresponding constraints explained above.

\subsubsection{Singular solitons (type-II)}

The last kind of soliton to be study for the case of Kerr law nonlinearity is the second type of singular soliton solution. In order to proceed, we first substitute the ansatz (78) into the real part equation (90), generating

$$
\begin{aligned}
& \left\{\left[\kappa\left(\alpha_{l}+b_{l} \omega-a_{l} \kappa\right)-\omega\right]-2 p^{2}\left(a_{l}-b_{l} \nu\right) B^{2}\right\} A_{l} \operatorname{coth}^{p} \tau \\
& \quad+p(p-1)\left(a_{l}-b_{l} \nu\right) A_{l} B^{2} \operatorname{coth}^{p-2} \tau \\
& \quad+p(p+1)\left(a_{l}-b_{l} \nu\right) A_{l} B^{2} \operatorname{coth}^{p+2} \tau \\
& \quad+\left[c_{l}+\kappa\left(\xi_{l}+\lambda_{l}\right)\right] A_{l}^{3} \operatorname{coth}^{3 p} \tau \\
& \quad-\operatorname{coth}^{p} \tau \sum_{n=1}^{N} \delta_{l n} A_{n}=0 .
\end{aligned}
$$

The coefficient of the stand alone element $\operatorname{coth}^{p_{l}-2} \tau$ as well as the balancing principle yield $p=1$. By substituting the resulting value of $p$ into (100) leads to the same results as for dark solitons (95)-(96), altogether with corresponding conditions. Therefore, the type-II singular soliton solution for the nonlinear system (89) is

$$
q^{(l)}(x, t)=A_{l} \operatorname{coth}[B(x-\nu t)] \mathrm{e}^{\mathrm{i}(-\kappa x+\omega t+\Theta)}
$$

where parameters and corresponding constraints are the same as for dark soliton in the case of multiple-core cou- 
plers when coupling with the all neighbors with Kerr law nonlinearity.

\subsection{Power law nonlinearity}

The last nonlinearity to be considered for core couplers coupling with all neighbors is power law. Under this nonlinearity the system (88) takes the new form

$$
\begin{aligned}
& \mathrm{i} q_{t}^{(l)}+\mathrm{i} \alpha_{l} q_{x}^{(l)}+a_{l} q_{x x}^{(l)}+b_{l} q_{x t}^{(l)}+c_{l}\left|q^{(l)}\right|^{2 n} q^{(l)} \\
& \quad+\mathrm{i} \Gamma_{l} q_{x x x}^{(l)}+\mathrm{i} \lambda_{l}\left(\left|q^{(l)}\right|^{2 n} q^{(l)}\right)_{x}+\mathrm{i} \xi_{l}\left|q^{(l)}\right|^{2 n} q_{x}^{(l)} \\
& \quad+\mathrm{i} \eta_{l}\left(\left|q^{(l)}\right|^{2 n}\right)_{x} q^{(l)}=\sum_{n=1}^{N} \delta_{l n} q^{(n)}
\end{aligned}
$$

The substitution of the guess hypothesis (61) into (101) leads to retrieve the same imaginary part we got on (48), consequently the speed $\nu$ as in (8) whenever the conditions (9) and (49) are satisfied. The resulting real part can be rewritten as

$$
\begin{aligned}
& a_{l} \frac{\partial^{2} P_{l}}{\partial x^{2}}+b_{l} \frac{\partial^{2} P_{l}}{\partial x \partial t}+\left[\kappa\left(\alpha_{l}+b_{l} \omega-a_{l} \kappa\right)-\omega\right] P_{l} \\
& +\left[c_{l}+\kappa\left(\xi_{l}+\lambda_{l}\right)\right] P_{l}^{2 n+1}=\sum_{n=1}^{N} \delta_{l n} P_{n} .
\end{aligned}
$$

The equation (102) will be further split into four subsections depending on the type of soliton that is being considered.

\subsubsection{Bright solitons}

To investigate the bright soliton dynamics on the system (101), we begin by inserting the waveform (65) into (102), yielding

$$
\begin{aligned}
& \left\{\left[\kappa\left(\alpha_{l}+b_{l} \omega-a_{l} \kappa\right)-\omega\right]+p^{2}\left(a_{l}-b_{l} \nu\right) B^{2}\right\} A_{l} \operatorname{sech}^{p} \tau \\
& \quad-p(1+p)\left(a_{l}-b_{l} \nu\right) A_{l} B^{2} \operatorname{sech}^{p+2} \tau \\
& \quad+\left[c_{l}+\kappa\left(\xi_{l}+\lambda_{l}\right)\right] A_{l}^{2 n+1} \operatorname{sech}^{(2 n+1) p} \tau \\
& \quad-\left(\sum_{n=1}^{N} \delta_{l n} A_{n}\right) \operatorname{sech}^{p} \tau=0 .
\end{aligned}
$$

Balancing principle allows one to compare dispersion and nonlinearity in $(103)$, thus $(2 n+1) p=p+2$ from which we can obtain the value of the parameter $p$ as in (83). The substitution of the resulting value of $p$ into (103), and setting the coefficients of the linearly independent functions $\operatorname{sech}^{j} \tau$ to zero generates the speed as in (84), and the wave number

$$
\begin{aligned}
\omega & =\left[(n+1)\left(\sum_{n=1}^{N} \delta_{l n} A_{n}\right)-\Gamma_{l} A_{l}^{2 n+1}\right. \\
& \left.-(n+1) \kappa\left(\alpha_{l}-a_{l} \kappa\right) A_{l}\right] /(n+1)\left(b_{l} \kappa-1\right) A_{l},
\end{aligned}
$$

where for convenience we defined $\Gamma_{l}=c_{l}+\kappa\left(\lambda_{l}+\xi_{l}\right)$. Next, the comparison of the two possible expressions of the soliton speed $\nu,(8)$ and (84) yields

$$
B=\sqrt{\frac{n^{2}\left(b_{l} \kappa-1\right)^{2} \Gamma_{l} A_{l}^{2 n+1}}{(n+1)\left\{\left(a_{l}-b_{l} \alpha_{l}\right) A_{l}+b_{l}^{2}\left(\sum_{n=1}^{N} \delta_{l n} A_{n}\right)\right\}-b_{l}^{2} \Gamma_{l} A_{l}^{2 n+1}}}(105)
$$

as long as the radicand stay positive. Therefore the bright 1-soliton solution is given by

$$
q^{(l)}(x, t)=A_{l} \operatorname{sech}^{\frac{1}{n}}[B(x-\nu t)] \mathrm{e}^{\mathrm{i}(-\kappa x+\omega t+\Theta)}
$$

where the association between the amplitudes and the inverse width is depicted in (105), the soliton speed can be consider either as (8) or (84), and the waves number is defined in (104). The corresponding constraints have to be satisfies in order for the soliton to propagate within the system (101).

\subsubsection{Dark solitons}

In the case of dark soliton the starting hypothesis is as in (70). Thus, the substitution of such hypothesis in (81) prompt to

$$
\begin{aligned}
& \{[ \\
& \left.\left.\quad \kappa\left(\alpha_{l}+b_{l} \omega-a_{l} \kappa\right)-\omega\right]-2 p^{2}\left(a_{l}-b_{l} \nu\right) B^{2}\right\} A_{l} \tanh ^{p} \tau \\
& \quad+p(p-1)\left(a_{l}-b_{l} \nu\right) A_{l} B^{2} \tanh ^{p-2} \tau \\
& \quad+p(p+1)\left(a_{l}-b_{l} \nu\right) A_{l} B^{2} \tanh ^{p+2} \tau \\
& \quad+\left[c_{l}+\kappa\left(\xi_{l}+\lambda_{l}\right)\right] A_{l}^{2 n+1} \tanh ^{(2 n+1) p} \tau \\
& \quad-\left(\sum_{n=1}^{N} \delta_{l n} A_{n}\right) \tanh ^{p} \tau=0 .
\end{aligned}
$$

The existing balance between dispersion and nonlinearity leads to the value of the parameter $p=1 / n$. However, the standalone element $\tanh ^{p-2} \tau$ leads to $p=1$, therefore $n=1$ implying $p=1$. As a direct consequence, the system with power law nonlinearity (101) collapses the system with Kerr law (89) when dealing with dark solitons, whose results were discussed in (94)-(96).

\subsubsection{Singular solitons (type-I)}

Now we proceed to discuss the last singular soliton type-I of this manuscript. To explore singular type-I soliton propagation on the system (101), we evaluate the hypothesis (75) within the real part equation (102), yielding

$$
\begin{aligned}
& \{[ \\
& \left.\left.\quad+\left(\alpha_{l}+b_{l} \omega-a_{l} \kappa\right)-\omega\right]+p^{2}\left(a_{l}-b_{l} \nu\right) B^{2}\right\} A_{l} \operatorname{csch}^{p} \tau \\
& \quad+p(1+p)\left(a_{l}-b_{l} \nu\right) A_{l} B^{2} \operatorname{csch}^{p+2} \tau \\
& \quad+\left[c_{l}+\kappa\left(\xi_{l}+\lambda_{l}\right)\right] A_{l}^{2 n+1} \operatorname{csch}^{(2 n+1) p} \tau \\
& \quad-\left(\sum_{n=1}^{N} \delta_{l n} A_{n}\right) \operatorname{csch}^{p} \tau=0 .
\end{aligned}
$$

Balancing principle leads the same value of $p=1 / n$. From the linearly independent functions one can retrieve the speed (87) while the wave numbers has the new form

$$
\omega=\frac{(n+1)\left(\sum_{n=1}^{N} \delta_{l n} A_{n}\right)+\Gamma_{l} A_{l}^{2 n+1}-(n+1) \kappa(\alpha-a \kappa)}{(n+1)\left(b_{l} \kappa-1\right) A_{l}},
$$

where $\Gamma_{l}=c_{l}+\kappa\left(\xi_{l}+\lambda_{l}\right)$. Equating the soliton speeds (8) with (87) leads to

$$
B=\sqrt{\frac{n^{2}\left(b_{l} \kappa-1\right)^{2} \Gamma_{l} A_{l}^{2 n+1}}{(n+1)\left[\left(a_{l}-b_{l} \alpha_{l}\right) A_{l}+b_{l}^{2}\left(\sum_{n=1}^{N} \delta_{l n} A_{n}\right)\right]+b_{l}^{2} \Gamma_{l} A_{l}^{2 n+1}}}
$$


subject to

$$
\begin{aligned}
& \Gamma_{l} A_{l}^{2 n+1}\left\{(n+1)\left[\left(a_{l}-b_{l} \alpha_{l}\right) A_{l}+b_{l}^{2}\left(\sum_{n=1}^{N} \delta_{l n} A_{n}\right)\right]\right. \\
& \left.+b_{l}^{2} \Gamma_{l} A_{l}^{2 n+1}\right\}>0 .
\end{aligned}
$$

Therefore the singular type-I soliton solution to (101) is

$$
q^{(l)}(x, t)=A_{l} \operatorname{csch}^{\frac{1}{n}}[B(x-\nu t)] \mathrm{e}^{\mathrm{i}(-\kappa x+\omega t+\Theta)}
$$

where the amplitude-inverse width relation, speed, and wave number are presented above together with corresponding solvability conditions.

\subsubsection{Singular solitons (type-II)}

The last type of soliton to be considered for power law nonlinearity is the singular type-II soliton. In order to study the dynamics of such soliton within the system (101) we substitute a hypothesis of the form (78) in the real part portion (102) to get

$$
\begin{aligned}
& \left\{\left[\kappa\left(\alpha_{l}+b_{l} \omega-a_{l} \kappa\right)-\omega\right]-2 p^{2}\left(a_{l}-b_{l} \nu\right) B^{2}\right\} A_{l} \operatorname{coth}^{p} \tau \\
& \quad+p(p-1)\left(a_{l}-b_{l} \nu\right) A_{l} B^{2} \operatorname{coth}^{p-2} \tau \\
& \quad+p(p+1)\left(a_{l}-b_{l} \nu\right) A_{l} B^{2} \operatorname{coth}^{p+2} \tau \\
& \quad+\left[c_{l}+\kappa\left(\xi_{l}+\lambda_{l}\right)\right] A_{l}^{2 n+1} \operatorname{coth}^{(2 n+1) p} \tau \\
& \quad-\left(\sum_{n=1}^{N} \delta_{l n} A_{n}\right) \operatorname{coth}^{p} \tau=0 .
\end{aligned}
$$

Once again, the balance between dispersion and nonlinearity leads to $p=1 / n$. Moreover, the standalone element $\operatorname{coth}^{p-2} \tau$ leads to $p=1$. Thus, equating both values of $p$ makes $n=1$. Therefore, for the type-II singular soliton solution, the system with power law nonlinearity (101) reduces to the system with Kerr nonlinearity (89), whose results, as in the case of dark soliton, were discussed above through (95)-(96) together with the corresponding constraints. Finally, the type-II singular soliton solution for the nonlinear system (63) has the form

$$
q^{(l)}(x, t)=A_{l} \operatorname{coth}[B(x-\nu t)] \mathrm{e}^{\mathrm{i}(-\kappa x+\omega t+\Theta)}
$$

where parameters along with corresponding constraints are the same as for dark soliton in the case of multiplecore couplers when coupling with the nearest neighbors with Kerr law nonlinearity.

\section{Conclusions}

This paper obtained perturbed bright, dark and singular soliton solutions for optical couplers by the method of undetermined coefficients. Two forms of nonlinearity are studied in this paper and they are Kerr law and power law. Both twin core as well as multiple core couplers are addressed. The multiple core couplers are further classified. When the coupling is with immediate neighbors and the second case is when coupling is with all neighbors. In all of these three forms of couplers, it has been established as usual that for power law nonlinearity, dark solitons will exist provided its nonlinearity parameter condenses to unity; in other words power law nonlinearity collapses to Kerr law. In fact, this is also true for the second form of singular optical solitons. These are phenomenal observations that are made in fibers, couplers and other optical devices. The paper will be extended further, later on, with additional form of nonlinearities and these are parabolic law, dual-power law, quadratic-cubic law, anticubic law and others. The results will be soon reported.

\section{References}

[1] A.A. Alshaery, E.M. Hilal, M.A. Banaja, S.A. Alkhateeb, L. Moraru, A. Biswas, J. Optoelectron. Adv. Mater. 16, 75 (2014).

[2] A.H. Arnous, M.Z. Ullah, S.P. Moshokoa, Q. Zhou, H. Triki, M. Mirzazadeh, A. Biswas, Nonlin. Dyn. 88, 1891 (2017).

[3] A. Bahrami, A. Rostami, F. Nazari, Optik 122, 1787 (2011).

[4] M.A. Banaja, S.A. Alkhateeb, A.A. Alshaery, E.M. Hilal, J. Vega-Guzman, Q. Zhou, A. Biswas, J. Computat. Theor. Nanosci. 13, 4660 (2016).

[5] G.P. Bava, G. Ghione, J. Math. Phys. 25, 1900 (1984).

[6] A. Biswas, J. Nonlin. Opt. Phys. Mater. 10, 329 (2001).

[7] A. Biswas, Fiber Integr. Opt. 20, 571 (2001).

[8] A. Biswas, J. Electromagn. Waves Appl. 15, 1189 (2001).

[9] A. Biswas, Opt. Quant. Electron. 35, 221 (2003).

[10] A. Biswas, J. Nonlin. Opt. Phys. Mater. 12, 45 (2003).

[11] A. Biswas, J. Nonlin. Opt. Phys. Mater. 13, 81 (2004).

[12] A. Biswas, S. Konar, Introduction to Non-Kerr Law Optical Solitons, CRC Press, Boca Raton (FL) 2006.

[13] A. Biswas, D.A. Lott, B. Sutton, K.R. Khan, M.F. Mahmood, J. Electromagn. Waves Appl. 27, 1976 (2013)

[14] H. Li, C. Xu, C. Tian, W. Wu, F. Xu, B. Yu, Optik 127, 694 (2016).

[15] M. Mirzazadeh, M. Eslami, Q. Zhou, M.F. Mahmood, E. Zerrad, A. Biswas, M. Belic, J. Nonlin. Opt. Phys. Mater. 24, 1550017 (2015).

[16] M. Mirzazadeh, M. Eslami, E. Zerrad, M.F. Mahmood, A. Biswas, M. Belic, Nonlin. Dyn. 81, 1933 (2015).

[17] M.M.A. Qurashi, E. Ates, M. In, Optik 142, 343 (2017).

[18] H. Saini, Optik 124, 3146 (2013).

[19] M. Savescu, A.H. Bhrawy, A.A. Alshaery, E.M. Hilal, K.R. Khan, M.F. Mahmood, A. Biswas, J. Mod. Opt. 61, $442(2014)$.

[20] B. Vakili, S. Bahadori-Haghighi, R. Ghayour, Optik 124, 6292 (2017). 